
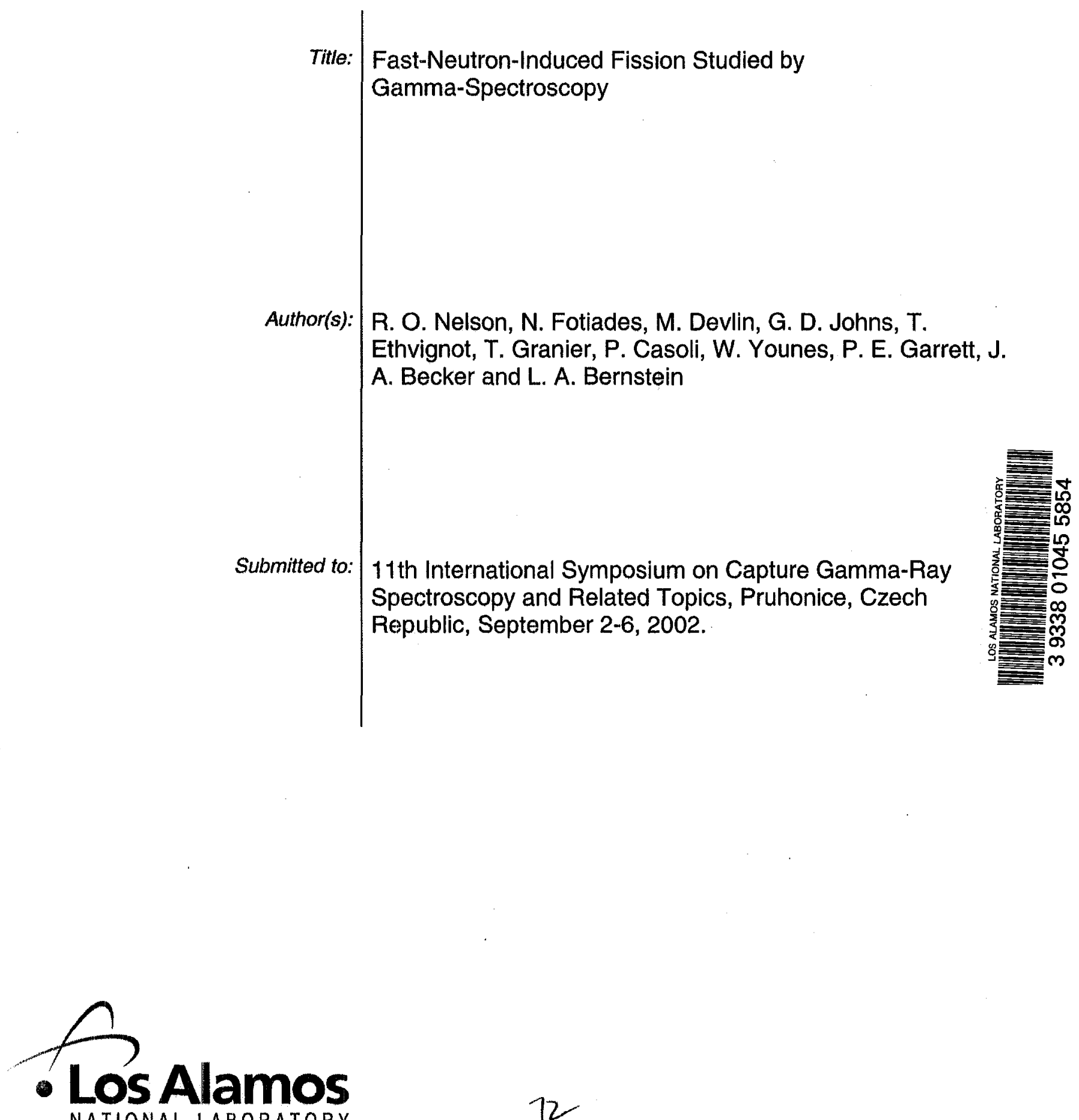

NATIONAL LABORATORY

Los Alamos National Laboratory, an affirmative action/equal opportunity employer, is operated by the University of California for the U.S. Department of Energy under contract W-7405-ENG-36. By acceptance of this article, the publisher recognizes that the U.S. Government retains a nonexclusive, royalty-free license to publish or reproduce the published form of this contributio $\equiv$ o allow others to do so, for U.S. Government purposes. Los Alamos National Laboratory requests that the publisher identify this article a $=$ k performed under the auspices of the U.S. Department of Energy. Los Alamos National Laboratory strongly supports academic rreedom and a researcher's right to publish; as an institution, however, the Laboratory does not endorse the viewpoint of a publication or guarantee its technical correctness. 


\title{
FAST-NEUTRON-INDUCED FISSION STUDIED BY GAMMA-SPECTROSCOPY
}

\author{
R. O. NELSON, N. FOTIADES, M. DEVLIN, G. D. JOHNS \\ LANSCE-3, MS H855, \\ Los Alamos National Laboratory, \\ Los Alamos, NM 87545, USA \\ E-mail: rnelson@lanl.gov \\ T. ETHVIGNOT, T. GRANIER, P. CASOLI \\ Commissariat à l'Énergie Atomique, BP 12, \\ 91680 Bruyères-le-Châtel, France \\ E-mail: thierry.ethvignot@cea.fr
}

\author{
W. YOUNES, P. E. GARRETT, J. A. BECKER AND L. A. BERNSTEIN \\ P.O. Box 808 \\ Lawrence Livermore National Laboratory, \\ Livermore, CA 94551 USA \\ E-mail: younes@llnl.gov
}

\begin{abstract}
Prompt gamma-ray and $\mathrm{x}$-ray spectroscopy techniques are being employed to study fast-neutron-induced fission of actinides to determine independent (pre-betadecay) yields for a wide range of product nuclides. Data are acquired using the GEANIE high-resolution gamma-ray spectrometer at the LANSCE/WNR unmoderated spallation neutron source providing neutrons with energies from below 1 $\mathrm{MeV}$ to over $400 \mathrm{MeV}$. Three different techniques (identification by characteristic gamma rays, by gamma-gamma coincidences, and by fission-gamma coincidences) are being used to gather complementary data sets from which detailed fission yields can be extracted. From these data, mass and charge distributions are determined over a wide incident-neutron energy range. The phenomena of interest include the transition from asymmetric to symmetric fission, the competition between neutron and gamma-ray emission, nuclear structure effects in fission and the angular momentum inparted to the fission products. Results for ${ }^{238} \mathrm{U}$ and ${ }^{235} \mathrm{U}$ are presented.
\end{abstract}

\section{Introduction}

There is renewed interest in neutron-induced fission, in particular at higher energies due to the potential use of high-energy accelerators for nuclear waste transmutation and energy generation, for the production of beams 
of rare and radioactive ions, to test models of fission using the increased computational power available ${ }^{1}$, and to learn more about the details of the fission process. Following a brief overview of some previous research, we describe the GEANIE gamma-ray spectrometer and some of the current studies of neutron-induced fission, including the determination of independent yields of nuclides and mass and charge distributions of primary fission products.

Fission products have been studied using gamma-ray spectroscopy for many years. A comprehensive review of post-fission phenomena that describes much of our knowledge of mass and charge distributions and other quantities is given by Hoffman and Hoffman. ${ }^{2}$ Cheifetz et al. performed one of the first experiments to use high-resolution gamma spectroscopy to study independent fission yields. ${ }^{3}$ Here we give a brief overview of some of the previous research on fission.

Spontaneous fission of ${ }^{252} \mathrm{Cf}$ and ${ }^{248} \mathrm{Cm}$ recently have been investigated with the large high-resolution gamma-ray spectrometers, Gammasphere and Euroball. Such measurements have provided excellent low-background data where rare processes such as alpha ternary fission have been observed, ${ }^{4}$ and properties of neutron-rich nuclei have been measured. ${ }^{5}$ However, such studies are limited to very few fissioning nuclei and essentially a single energy.

Charged particle-induced fission has been studied using protons, deuterons and other ions. These studies probe fission at higher energies and have provided the majority of the data at intermediate energies. The use of heavy ions brings in large angular momentum allowing the study of fission under more extreme conditions.

Previous studies of neutron-induced fission have mostly been conducted at incident neutron energies below $15 \mathrm{MeV}$ due to limitations of neutron sources. The majority of neutron-induced fission data comes from thermal neutrons, reactor fast-neutron spectra, $14-\mathrm{MeV}$ neutrons, and some data taken with monoenergetic neutron sources typically below $10 \mathrm{MeV}$.

The fission process is described as elongation of the nucleus followed by scission in which neutron-rich primary fragments are emitted. These fragments rapidly emit neutrons after which we refer to them as primary products. About $10 \%$ of the primary products that we observe in gammadecay studies are stable, while the other $90 \%$ undergo beta decay on a time scale of ms to 100's of seconds. It is primarily the prompt gamma-rays emitted in less than $10 \mathrm{~ns}$ from the primary products that are measured in our experiments. From the prompt gamma-ray yields we can infer fission product yields for many product nuclides including the stable products that 
are not usually observed in radiochemical measurements of fission yields. In addition we have measured $\mathrm{K} \mathrm{x}$-ray yields from the primary products which allow us to deduce charge distributions (yields summed over isotopes) and which complement the data obtained from the gamma rays.

At incident neutron energies below the neutron binding energy $(\sim 6$ $\mathrm{MeV}$ for actinides) only fission of the $\mathrm{A}+1$ compound nucleus is possible. However, at higher energies one or more prefission neutrons may be emitted in $(\mathrm{n}, \mathrm{xn} \mathrm{f})[\mathrm{x}=1,2,3, \ldots]$ reactions. Thus at higher energies the observed yields are summed over more than one fissioning system (e.g. ${ }^{239} \mathrm{U},{ }^{238} \mathrm{U}$, ${ }^{237} \mathrm{U}$...).

It is estimated that about one thousand different product nuclei are created in the fission of actinide nuclei, although many are produced only with very low probablity. Each of these product nuclei can emit several gamma rays thus producing a very complicated spectrum. The product yields range over many orders of magnitude with the maximum yield being about $4 \%$ of the total fission yield. In our gamma-ray measurements we observe approximately 100 different product nuclei representing about $10 \%$ of the different nuclides produced, but accounting for almost $90 \%$ of the total fission yield.

\section{Experiment}

The GEANIE high-resolution photon detector array ${ }^{6}$ and the LANSCE/WNR broad-spectrum spallation neutron source have been described elsewhere. ${ }^{7}$ In these measurements 11 LEPS, for x-ray and low-energy gamma rays, and 15 coaxial HPGe detectors, for higher-energy gamma rays, were used. BGO escape-suppression shields are used on a majority of the Ge detectors. The energies of the incident neutrons is determined by the time-of-flight technique. For the singles gamma-ray and gamma-gamma coincidence measurements, samples consisted of $\sim 400 \mathrm{mg} / \mathrm{cm}^{2}$ metal disks of ${ }^{238} U$ (99.8\% enriched) and ${ }^{235} U$ (93.15\% enriched).

For the x-ray measurements, however, it was necessary to detect the fission fragments in coincidence with the $x$-rays to get a clean spectrum in which the x-rays were observable above background. For these measurements solar cells were used as fission fragment detectors. ${ }^{8}$ A total of 8 solar cells with thin $\left(\sim 1 \mathrm{mg} / \mathrm{cm}^{2}\right){ }^{238} \mathrm{U}$ deposits were used. A schematic diagram of the active target is shown in Fig. 1. Very thin $(\sim 75 \mathrm{~mm})$ Si solar cells were used to reduce attenuation of $x$-rays in the target. The active target was modeled with $\mathrm{MCNP}^{9}$ to calculate the attenuation in the sample. The transmission averaged over the 8 cells ranged from about $20 \%$ for $10 \mathrm{keV}$ $\mathrm{x}$-rays (light products) to $70 \%$ for $35-\mathrm{keV} \mathrm{x}$-rays (heavy products). 


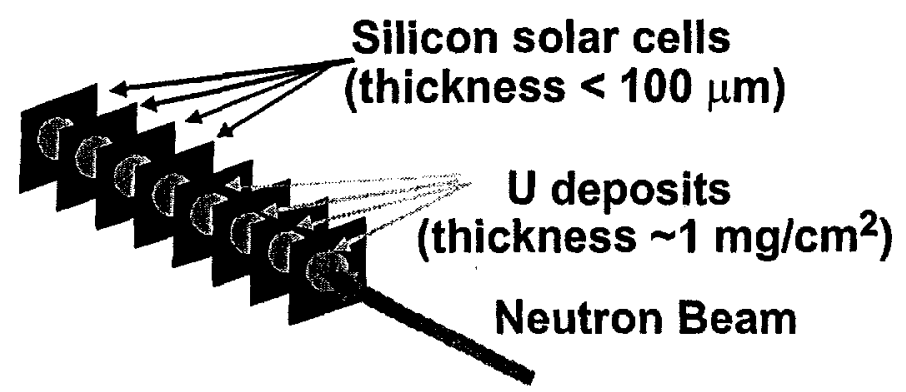

Figure 1. Schematic diagram of the solar cells used to detect the fission fragments in coincidence with the $\mathrm{x}$-rays.

\section{Analysis and Results}

\section{1. ${ }^{235} U(n, f)$}

Results from a study of single gamma-rays and limited gamma-gamma coincidence data have been published. ${ }^{10}$ Over 200 gamma rays from 56 fission products were observed, and excitation functions were obtained for 146 gamma rays. However, for the purpose of analyzing the transition from asymmetric to symmetric fission, only 29 clean transitions from 22 fission products that were within one standard deviation of a Gaussian fit to the evaluated $14-\mathrm{MeV}$ data of James et al. ${ }^{11}$ were used. The observed mass distributions were fit with a 5-Gaussian-curve model in order to deduce the symmetric and asymmetric contributions.

A major goal of this study was to examine the transition from asymmetric to symmetric fission in ${ }^{235} \mathrm{U}$ over a wide energy range. This transition can be interpreted as an indication of the disappearance of shell effects at high-excitation energies in the fissioning system. The result observed is a change from predominantly asymmetric fission to predominantly symmetric fission over a range of about $20 \mathrm{MeV}$ in excitation energy, starting at a probability for symmetric fission of $10 \%$ around $14 \mathrm{MeV}$ and reaching $90 \%$ by $35 \mathrm{MeV}$. These data are unique for neutron-induced fission and in having continuous coverage over a large energy range.

\section{2. ${ }^{238} U(n, f)$}

Gamma-ray measurements and gamma-gamma coincidence measurements have been made on ${ }^{238} \mathrm{U}$, but here we concentrate on recent mesaurements of the $\mathrm{x}$-rays from fission products. The $\mathrm{x}$-rays following fission are produced primarily by electrons filling orbitals following internal conversion. Thus greater $\mathrm{x}$-ray yields (up to $1 \mathrm{x}$-ray per product nuclide) are observed for 
the higher $\mathrm{Z}$ (heavier) products. The $\mathrm{x}$-ray yields depend strongly on the structure of the low-lying nuclear levels and the energy and multi-polarity of the transitions. Strong odd-even mass effects are observed. Detailed fitting of the $K_{\alpha}$ and $K_{\beta}$ x-rays was carried out to extract the total $x$-ray yields for a given element. A portion of the $\mathrm{x}$-ray spectrum from the heavy-mass products is shown in Fig. 2.

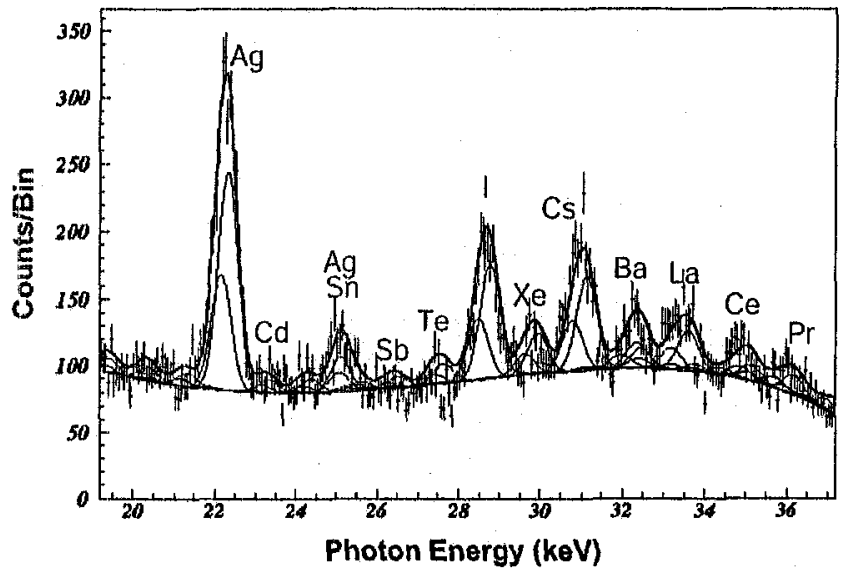

Figure 2. Spectrum of $x$-rays for some of the heavier-mass fission products observed with GEANIE. Peaks are labelled with the symbol of the element from which the $x$-rays are emitted. The lines are fits to the $K_{\alpha}$ and $K_{\beta} x$-ray lines and background. The large silver peaks are due mainly to inelastic excitation of nuclei in the silver contact on the solar cell.

We use the prescription of Reisdorf et al. ${ }^{13}$ that was obtained from detailed measurements of thermal-neutron-induced fission of ${ }^{233} \mathrm{U},{ }^{235} \mathrm{U}$, and ${ }^{252} \mathrm{Cf}$ spontaneous fission to relate measured $\mathrm{x}$-ray yields to charge yields. Due to the shift of fission products to lighter isotopes with increasing incident neutron energy (with increased neutron emission) $x$-ray yields are expected to change somewhat with increasing incident neutron energy. This change will be greater for the light-mass fragments than for the heavymass fragments due to the greater shift in masses observed for the light fragment peak. We observe average mass shifts that are typically 1 or 2 mass units over a range of $400 \mathrm{MeV}$. We expect to be able to estimate these effects based on observed mass shifts from our gamma-ray data and 
on the known low-energy structure of the product nuclides. The preliminary charge yield results shown in Fig. 3 use the Residorf prescription ${ }^{13}$ without modification. Results for higher-energy neutrons $\left(50<\mathrm{E}_{n}<400 \mathrm{MeV}\right.$ ) show that the symmetric fission region of the charge distribution fills in and the distribution may broaden slightly.

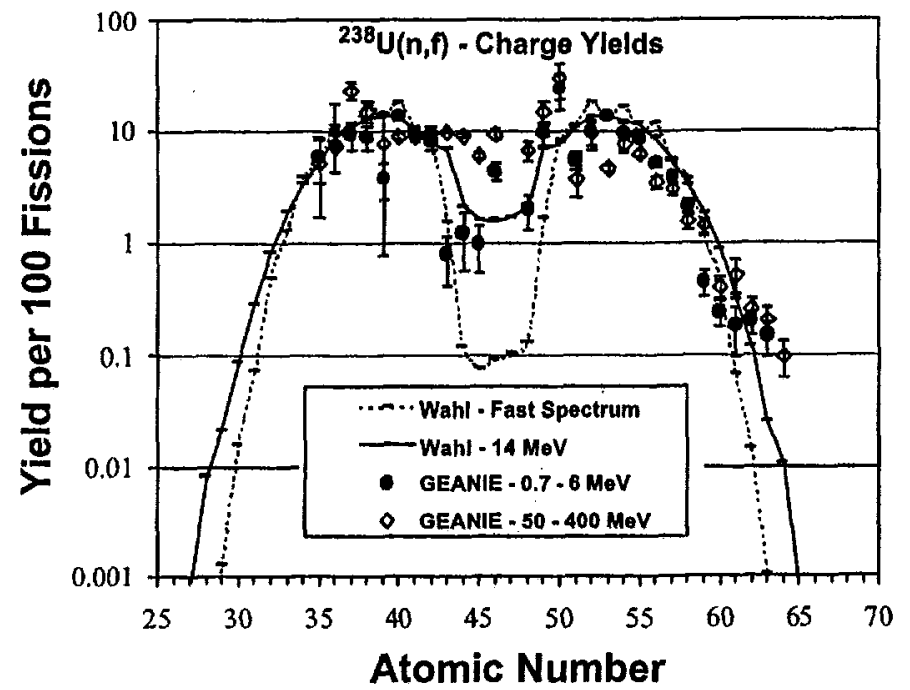

Figure 3. Preliminary charge yields deduced from the GEANIE x-ray data are compared with the systematics of $A$. Wahl, ${ }^{12}$ The GEANIE data are summed over the energy ranges $0.7<\mathrm{E}_{n}<6 \mathrm{MeV}$ and $50<\mathrm{E}_{n}<400 \mathrm{MeV}$. The lower curve is for a reactor neutron spectrum peaking near $\mathrm{E}_{n}=1 \mathrm{MeV}$ while the upper curve is for 14-MeV neutron-induced fission of ${ }^{238} \mathrm{U}$. Corrections for shifts in the mass distributions with energy remain to be done.

These results demonstrate that new information on neutron-induced fission can be obtained using a spallation neutron source and gamma-ray spectroscopy. The measured product yields, their observed gamma-ray spectra and the mass and charge distributions give information about the fission process that is important for a detailed understanding of fission as well as being useful for applications.

\section{Acknowledgments}

This work was funded by the US Department of Energy under contracts number W-7405-ENG-36 (LANL) and W-7405-ENG-48 (LLNL) and by the CEA/DAM Bruyères-le-Châtel. T. Ethvignot was partially supported by the Délégation Générale pour l'Armement of the French Ministry of De- 
fense. This work has benefited from the use of the Los Alamos Neutron Science Center at LANL. This facility is funded by the U.S. Department of Energy under Contract No. W-7405-ENG-36.

\section{References}

1. See for example, P. Moller, D. G. Madland, A. J. Sierk, and A. Iwamoto, Nature 409, 785 (2001).

2. D. C. Hoffman and M. M. Hoffman, Ann. Rev. Nucl. Sci. 24, 151 (1974).

3. E. Cheifetz, J. B. Wilhelmy, R. C. Jared, and S. G. Thompson, Phys. Rev. C4, 1913 (1971).

4. See for example, A. Ramayya, et al., Prog.in Part. and Nucl. Phys. 46, 221 (2001).

5. See for example, D. Patel, et al., J. Phys. G, 28, 649 (2002).

6. J. A. Becker and R. O. Nelson, Nucl. Phys. News Int. 7, 11 (1997).

7. P. W. Lisowski, C. D. Bowman, G. J. Russell, and S. A. Wender, Nucl. Sci. Eng. 106, 208 (1990).

8. T. Ethvignot, T. Granier, L. Giot, P. Casoli, and R. O. Nelson, Nucl. Instrum. Meth. A 490, 559 (2002).

9. J. Briesmeister, ed., "MCNP - A Monte Carlo N-Particle Transport Code", LANL Report, LA-12625-M (1997).

10. W. Younes, J. A. Becker, L. A. Bernstein, P. E. Garrett, C. A. McGrath, D. P. McNabb, R. O. Nelson, G. D. Johns, W. S. Wilburn, and D. M. Drake, Phys. Rev. C64, 054613 (2001).

11. M. F. James, R. W. Mills, and D. R. Weaver, Prog. Nucl. Energy 26, 1 (1991); M. James and R. Mills, Tech. Rep. AEA-TRS-1015, 1018 and 1019, UKAEA, BNF, PLC and Nuclear Electric, 1993 (unpublished) available from the National Nuclear Data Center at http://www.nndc.bnl.gov in the ENDF database, MAT \#9420, June 1993.

12. A. Wahl, in Compilation and Evaluation of Fission Yield Nuclear Data, IAEA-TECDOC-1168, 2000, p. 58.

13. W. Reisdorf, J. P. Unik, H. C. Griffin, and L. E. Glendenin, Nucl. Phys. A177, 337 (1971). 